\title{
A Challenging Management of Pseudomonas Perforated Corneal Ulcer with Multilayer Amniotic Membrane Transplantation and Pericardial Patch Graft in Pediatric Patient
}

\author{
Authors: \\ Nabilah Afifah*৫ \\ Herwindo Dicky Putranto \\ Lely Retno Wulandari ${ }^{\circ}$
}

\begin{abstract}
Affiliations:
Department of Ophthalmology, Faculty of Medicine, Universitas Brawijaya - RSUD Dr. Saiful Anwar Malang, Indonesia.
\end{abstract}

Corresponding author: Nabilah Afifah

bilaffh@student.ub.ac.id

\section{Dates:}

Received: 24 August 2021

Revised: 08 November 2021

Accepted: 10 November 2021

Published: 29 November 2021

DOI:

https://doi.org/10.20473/

vsehj.v1i1.2021.22-27

\section{Copyright:}

(C) 2021 Author(s). Open access under Creative Commons Attribution-Share Alike 4.0 International Licence (CC-BY-SA).

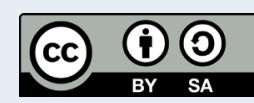

\begin{abstract}
Introduction: Pseudomonas aeruginosa ( $P$. aeruginosa) is the leading cause of corneal ulcers in children 0 to 3 years of age compared to children in general. Case presentation: A two-months- old infant presented with whitish patches on the right eye two days before admission. A central corneal ulcer with a size of 7-mm x 7-mm accompanied by corneal thinning and melting was shown on the right cornea. It is was surrounded by greyish white creamy infiltrates. Corneal scraping showed Pseudomonas aeruginosa specimens. The cornea became perforate and crystalline lens extrusion was found at the day after intravenous ceftriaxone, levofloxacin eye drop, and cefazoline fortified eye drop administering. It might be caused by bacterial elastase and toxin which contributed to corneal damage. The patient was underwent a multilayer Amniotic Membrane Transplantation (AMT) combined with a pericardial patch graft due to corneal perforation. Two months post-AMT and pericardial patch graft the corneal perforation became entirely heal due to multilayer AMT, despite lysis of the pericardial patch graft. Corneal scar formation and reduction of vitreous opacity in ultrasound examination were shown. The patient was planned to undergo keratoplasty. Conclusions: Corneal ulcers due to Pseudomonas aeruginosa are highly destructive. The levels of infection, diagnostic, and therapeutic are still problems in pediatric patients. Lens extrusion and lysis of the pericardial patch graft are examples in this case. Keratoplasty is the definitive treatment for corneal ulcers with perforation; however, multilayer AMT combined with pericardial patch graft can be used as an alternative therapy to accelerate wound healing, reduce inflammation, and maintain the integrity of the eyeball.
\end{abstract}

Keywords: Pseudomonas aeruginosa; pediatric corneal ulcer; amniotic membrane transplantation; pericardial patch graft

\section{Introduction}

Corneal ulcer is an infectious condition of the cornea that disrupts the epithelial layers involving the corneal stroma. Corneal ulcers are one of the leading causes of monocular blindness after untreated cataracts in many developing countries in Asia, Africa, and the Middle East. ${ }^{[1]}$

According to the World Health Organization ${ }^{[2]}$, approximately 700.000 children each year have a pathological condition of the cornea that permanently affects vision. The incidence of blindness due to keratitis in children is twenty times higher in the tropics and developing countries. Eye trauma is the leading predisposing cause of keratitis in children in $26-58 \%$ of cases. Several studies before 2000 showed that trauma (21.1-44.0\%), systemic disease (14.0-30\%), ocular surface disease (17.7-22.7\%), and history of surgery on the anterior segment (8.824.0\%) were the causes of keratitis. ${ }^{[3]}$ In addition, studies in the United States, India, and Taiwan ${ }^{[2],[4]}$ have shown that contact lens wear and environmental factors also play a role in causing keratitis.

The spectrum of micro-organisms causing corneal ulcers varies depending on geographic location. The most common cause of corneal ulceration is keratitis caused by bacteria, fungi, viruses, and protozoa, which is established 
based on clinical practice examination by supporting microbiological staining or culture of corneal tissue. Staphylococcus aureus and Streptococcus pneumoniae are gram-positive micro-organisms, and Pseudomonas aeruginosa ( $P$. aeruginosa) is a gram-negative microorganism mainly causing keratitis, especially in children. Pseudomonas aeruginosa and $\alpha$-hemolytic streptococci are the leading causes in children aged 0 to 3 compared to children in general, and the incidence in children $<3$ years of age is two times higher than in children of other ages. ${ }^{[2],[5]-[6]}$

Pseudomonas aeruginosa, Coagulase-negative staphylococci, and Staphylococcus aureus are the causes of contact lens-related keratitis in children. Pseudomonas aeruginosa is a gram-negative rod that thrives in moist environments such as water and soil. In children, Pseudomonas aeruginosa is an opportunistic pathogen associated with damage to the corneal tissue due to trauma, prematurity, and contact lens wear. The pathophysiology of Pseudomonas aeruginosa infection in children is influenced by virulence factors, namely glycocalyx, pili, flagella, endotoxins and exotoxins, and bacterial enzymes. These factors can interfere with protein synthesis and damage cell membranes. ${ }^{[7]}$

The goals of treating corneal ulcers in children are eradicating infection with antimicrobials, preventing scarring, and preventing visual disturbances. As opposed to adults, surgical management is rarely required in children except in impending perforation and corneal perforation. Surgical actions that can be done are cyanoacrylate glue, therapeutic penetrating or lamellar keratoplasty, conjunctival flap, debridement, and amniotic membrane transplantation. ${ }^{[6]}$

This case report discusses the detailed steps of examination, diagnosis, and medical and surgical management of Pseudomonas corneal perforation in pediatric patients.

\section{Case presentation}

A two-month-old infant presented with whitish patches on the right eye two days before admission. The patient complained about swollen, red, watery, and yellow discharge on his right eye on the first day. He was then brought to a general practitioner in the local community health center and was given a chloramphenicol eye ointment. The next day, the condition worsened with whitish patches on his right eye. The patient was then referred to and hospitalized in Dr. Saiful Anwar General Hospital.

The patient is the third child of three siblings with a history of the second child dying prematurely. Based on the mother's antenatal care (ANC) for monthly checkups to an obstetrician, there is no history of fever, sore throat, vaginal discharge, or infections in the genital area. There was no history of consuming alcohol, cigarettes, or drugs. The baby's delivery was through cesarean section at 36 weeks of gestation, with good condition, weighing 2.100 grams and immediately crying. The patient showed normal development and growth according to age, and he has completed basic immunization standards. There is no history of the same disease in the family and no history of trauma to the eye.

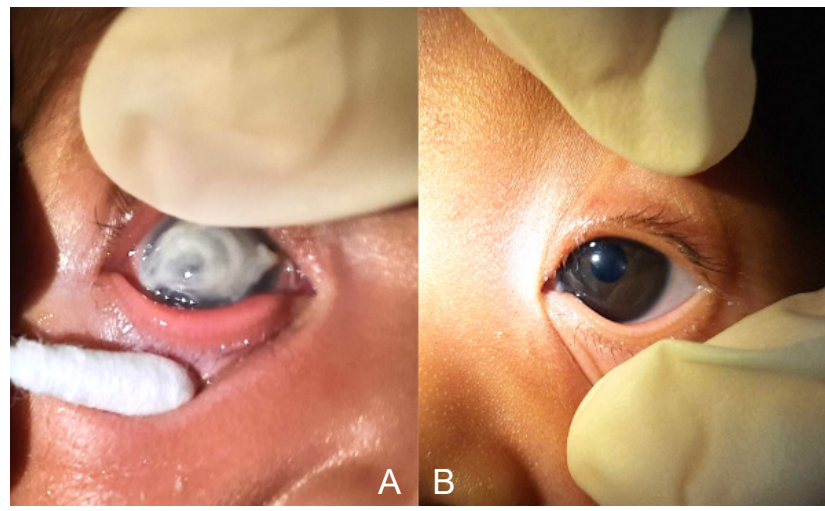

Figure 1. The patient's condition first day. The anterior segment on the first examination. Right eye (A) $7 \times 7 \mathrm{mmm}$ corneal ulcer, the central location of the cornea, gray with infiltrating, ring ulcer, melting, thinning. No scleral involvement. Left eye (B) within normal limits.

The patient's physical examination showed the vital signs within normal limits. The patient's body weight was 4.300 grams, and visual examination revealed no blink reflex on the right eye. Further examination of the anterior segment of the eye revealed hyperemic and edematous eyelids, yellow mucopurulent secretions, conjunctival injection, and chemosis, pericorneal injection, 7 x $7 \mathrm{~mm}$ corneal ulcer, the central location of the cornea, gray infiltrate, melting, thinning and there is no involvement of the sclera. The iris, lens, and pupil are challenging to evaluate. Intraocular pressure (IOP) by palpation revealed n-1 right eye (Figure 1 ).

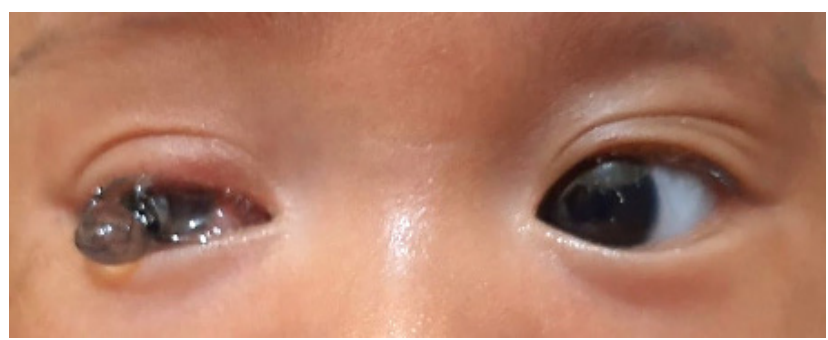

Figure 2. Two days after the admission. Lens prolapse was seen on the right eye.

Laboratory investigations showed a leukocyte count of $18.700 \times 103 / \mu \mathrm{L}$, and other complete blood counts were within normal limits. Hemostatic function, blood sugar, and electrolyte levels were within normal limits.

Based on the symptoms and the examination results, the patient was hospitalized with a diagnosis of severe corneal 
ulcer with a suspected bacterial infection. Administration of $2 \times 100 \mathrm{mg}$ intravenous ceftriaxone, levocin eyedrop of $6 \mathrm{gtts}$ on the right eye, fortified cefazolin of $1 \mathrm{gtt} / \mathrm{hour}$ on the right eye. Corneal scraping was performed for gram, Koh, and antibiotic sensitivity cultures.

Two days after the admission, an examination of the right eye revealed a spontaneous corneal perforation with lens prolapse. Palpebral hyperemia and chemosis, pericorneal injection, and mucopurulent secretions increased compared to the previous day (Figure 2).

Based on clinical findings, the patient was planned for keratoplasty; however, no available donor was found. Therefore, the patient was treated surgically with an amniotic membrane graft combined with a pericardial patch graft. First, we performed a 360-degree peritomy, then attached the three layers for the Amniotic Membrane Transplantation (AMT), made the shape and size of the pericardial graft according to the cornea, placed the pericardial patch graft over the surface of the AMT, and ended the procedure by suturing the conjunctiva covering the graft and applying chloramphenicol eye ointment (Figure 3). We have given $2 \times 100 \mathrm{mg}$ of intravenous ceftriaxone, $6 \times 1$ of levofloxacin eye drops, $6 \times 1$ of artificial tears eye drops, fortified cefazoline every hour, and $4 \mathrm{x}$ 1 of chloramphenicol eye ointment on the right eye. An experienced infection and immunology ophthalmologist carried out the surgery, and it was successful.

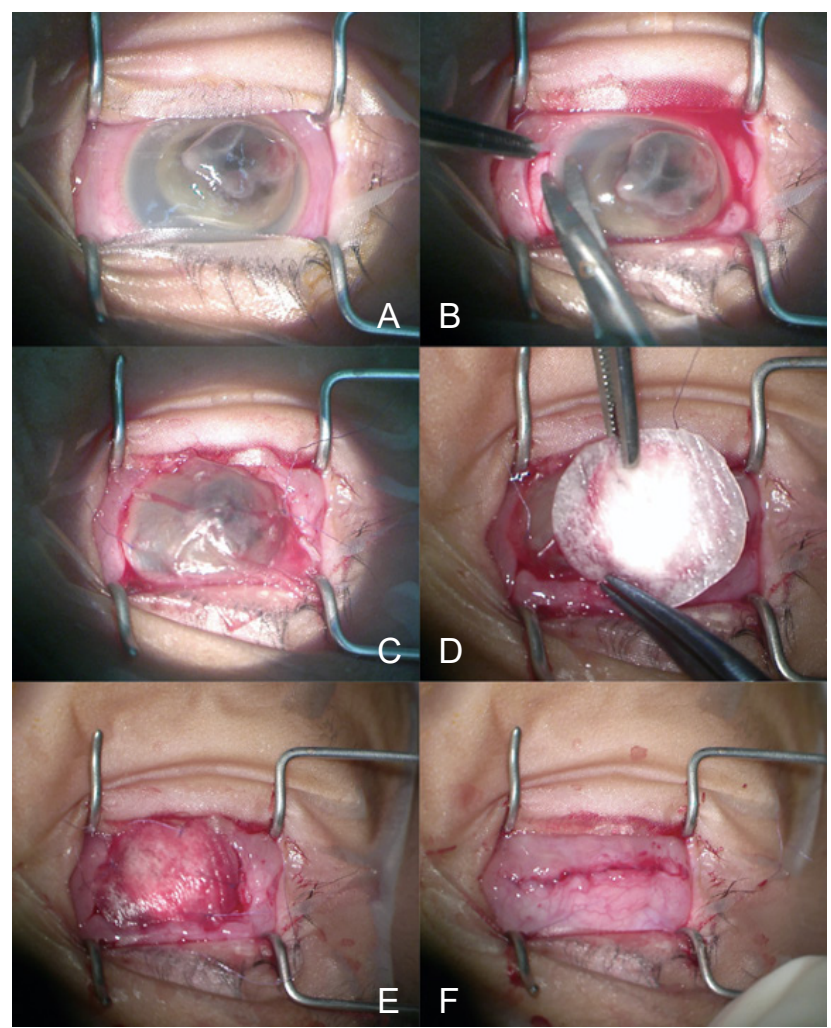

Figure 3. Amniotic Membrane Transplantation (AMT) and pericardial patch graft surgery techniques. (A) Anterior segment image of the right eye before surgery; (B) 360-degree peritomy; (C) Installation of three layers of AMT; (D) Make a shape and the size of the pericardial graft according to the cornea; (E) Placing the pericardial patch graft over the surface of the AMT; (F) Suturing the conjunctiva over the graft surface.

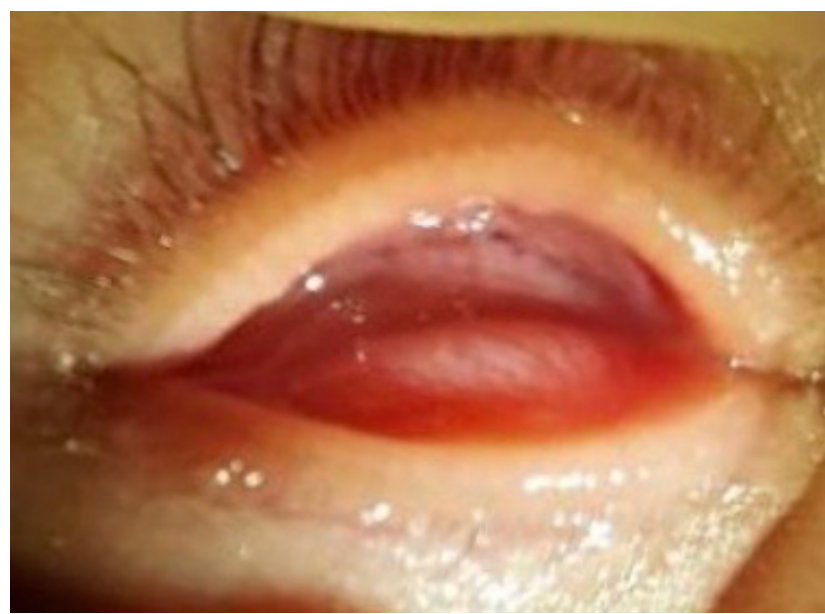

Figure 4. Three days after the admission. Anterior segment of the right eye on day one postoperative. Good condition of the conjunctival suture.

The day after surgery, an examination of the right eye revealed a tight and good condition of the conjunctival suture apposition (Figure 4). The patient continued his medical therapy. The gram stain, bacterial, and fungal culture results showed that $4+$ inflammatory cells indicated an inflammatory process in the sampling area, which may be due to aerobic or anaerobic bacteria, fastidious bacteria, and other non-infectious causes. Normal flora was taken at the time of taking the specimen. No morphology of hyphae, budding cells, and acanthamoeba was found; however, the culture showed gram-negative rods with 4+ inflammatory cells, Pseudomonas aeruginosa bacteria. The recommended antibiotics based on the culture are the aminoglycoside group, namely gentamicin $30 \mu \mathrm{g}$ and amikacin $30 \mu \mathrm{g}$; the fluoroquinolone group, namely ciprofloxacin and levofloxacin and; the cephalosporin group, namely ceftazidime. Ultrasound examination gave the impression of vitreous opacification with low to medium spike ec inflammatory cells and vitreous hemorrhage (Figure 5A). The patient was discharged on the next day.

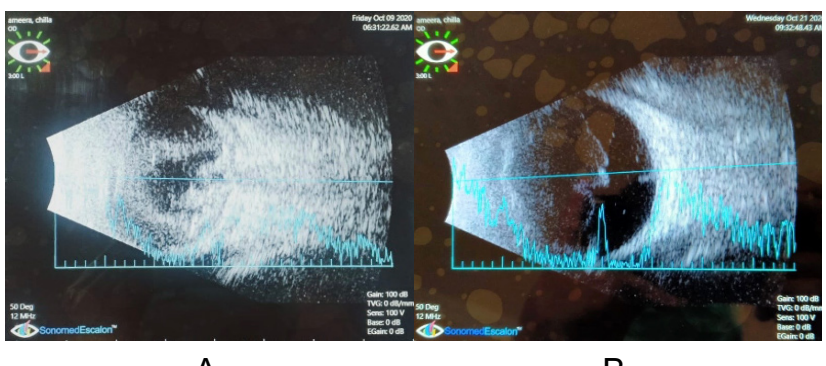

A

B

Figure 5. (A) Ultrasound examination of the right eye on the first postoperative day, the impression looks organized vitreous opacity with low to medium spike; (B) Ultrasound examination of the right eye on the thirteen day postoperatively, organized vitreous opacity with low to medium spike.

On the the thirteenth day, the patient returned to the eye clinic at Dr. Saiful Anwar General Hospital, Malang. 
The patient's parents complained about loose stitches in the right eye. The anterior eye segment examination revealed minimal eyelid edema and spasm, intact AMT; however, the pericardial patch graft experienced lysis (Figure 6A). Ultrasound examination gave the impression of vitreous opacification with low to medium spike ec inflammatory cells and vitreous hemorrhage (Figure 5B). The patient continued his medical therapy.

Two months post-AMT and pericardial patch graft, the patient has no complaints. On examination of the anterior segment of the eye, a corneal leucoma was seen (Figure 6B). Multilayer AMT completely healed corneal perforation despite lysis of the pericardial patch graft. Ultrasound examination showed improvement by reducing vitreous opacification with low to medium spike ec inflammatory cells and vitreous hemorrhage (Figure 7 ). The axial length of the right eye is 19.5. The patient continued the therapy with artificial tear eye drops and chloramphenicol eye ointment $6 \times 1$ on the right eye. The patient is planned for keratoplasty if a donor is available.

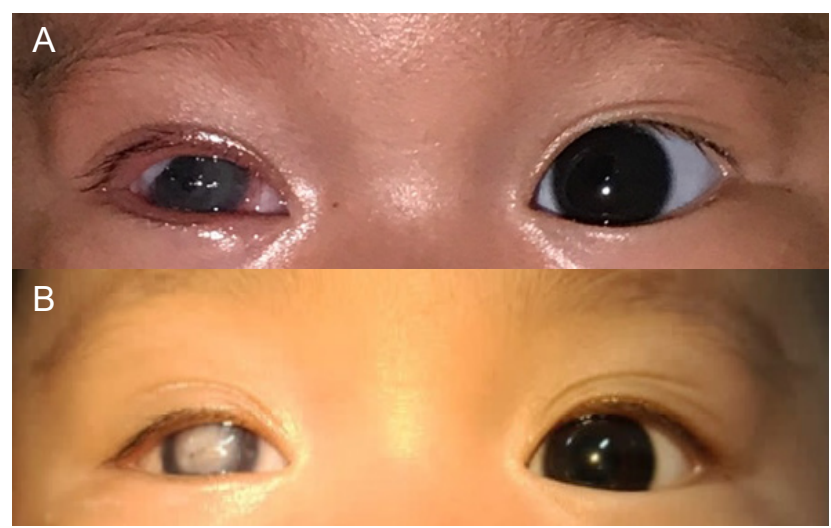

Figure 6. (A) Anterior segment view of the right eye on the thirteenth postoperative day intact AMT and lysis pericardial graft of seen; (B) Anterior segment view of the right eye a month after postoperative day, corneal leucoma is seen.

\section{Discussion and conclusions}

Corneal ulcer or ulcerative keratitis is an inflammatory or infectious condition of the cornea that involves disruption of the epithelial lining with involvement of the corneal stroma. Pseudomonas aeruginosa infection is common in young children. In children under three years of age, more than $50 \%$ of cases have been caused by Pseudomonas aeruginosa. Pseudomonas aeruginosa usually presents as a condition in which bacteria multiply rapidly, resulting in the inflammation of the epithelium with edema, ulceration of the stroma, and can cause significant stromal tissue damage, ending in loss of vision. ${ }^{[5],[8]}$

Pseudomonas aeruginosa is an aerobic gram-negative bacillus that grows in temperatures ranging from 30 $37^{\circ} \mathrm{C}$ and shows gray or greenish colonies. Infections caused by these organisms are generally fulminant. The pathophysiology of Pseudomonas aeruginosa is mediated by the host's Cystic Fibrosis Transmembrane Conductance Regulator (CFTR) and asialo-GM1. Bacterial elastase contributes to tissue damage directly and indirectly by activating host proteases. These bacteria secrete toxins, namely ExoU and ExoT, proteolytic enzymes. ExoU has been shown to kill macrophages and epithelial cells in vitro through its phospholipase activity. ExoT is an adenosine diphosphate ribosyltransferase that interferes with actin cytoskeletal regulation and inhibits phagocytosis, thereby increasing the survival of Pseudomonas aeruginosa. ${ }^{[6],[9]}$

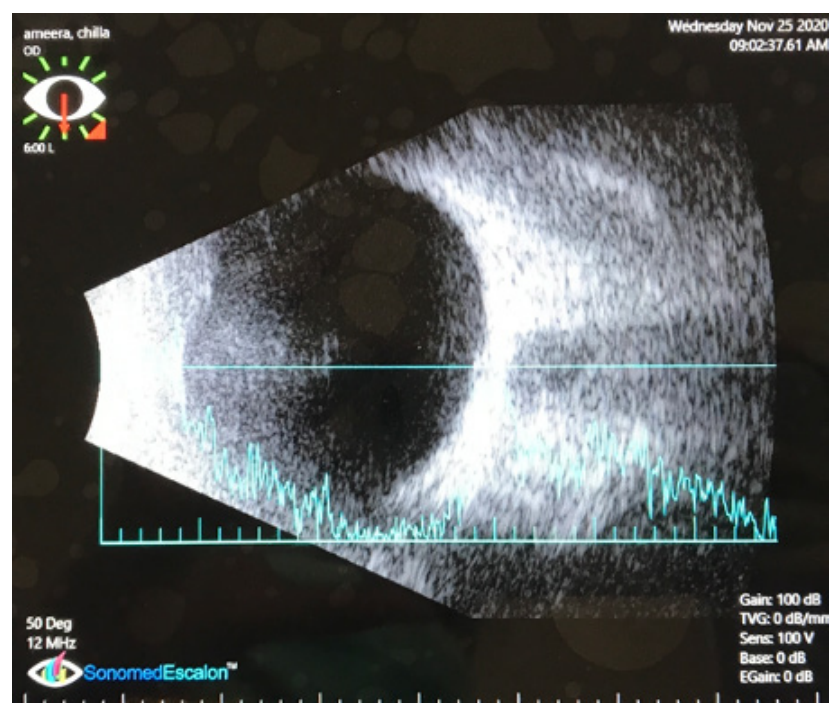

Figure 7. Ultrasound examination of the right eye showed improvement of the vitreous opacity a month after postoperative day,.

The clinical manifestations of corneal ulcers vary widely, including red eyes, pain, decreased vision, tearing, photophobia, discharge, and swollen eyelids. The onset of fungal ulcers is quieter than Pseudomonas aeruginosa, which grows and develops rapidly. ${ }^{[6]}$ Slitlamp examination revealed a yellow or white suppurative infiltrate with epithelial and stromal defects. The hallmark of Pseudomonas aeruginosa ulcers is a diffuse white epithelium, and an epithelial-stromal infiltrate. Ring ulcers are often seen at 48 to 96 hours with an infection that is not treated and diagnosed quickly. Ring ulcers are ring-shaped accumulations of polymorphonuclear leukocytes surrounding the central corneal lesion and are characteristic of Pseudomonas aeruginosa. Progressive ulcers that are not treated properly can cause the cornea to melt with a greenish-yellow mucopurulent fluid that adheres to the ulcer-the results in a descmetocele and eventually perforation within 2 to 5 days after the onset of infection. Conjunctival chemosis is also a sign of Pseudomonas aeruginosa infection as a possible underlying cause. ${ }^{[6],[9],[10],[11]}$

In this case, the cause of corneal ulcers is challenging to establish if the reasoning is only based on clinical signs. However, it can be better established by history, 
onset, symptoms, and clinical signs according to the characteristics of the cause of corneal ulcers, especially Pseudomonas aeruginosa.

The management of corneal ulcers consists of medical and non-medical. The administration of broad-spectrum antibiotics is the primary therapy in ulcers caused by bacteria before getting the results of staining and culture. Topical antibiotics (monotherapy) may be given for deep stromal involvement or infiltrates larger than $2 \mathrm{~mm}$ in size. Monotherapy consists of topical fluoroquinolones, cephalosporins, or aminoglycosides every 1-2 hours. In cases of extensive infiltrates or severe infection in the presence of hypopyon, fortified antibiotics may be given. Systemic antibiotics are rarely required; however, may be considered in severe cases involving the limbus or sclera, impending perforation, perforation, and in children with an infectious etiology due to Haemophilus influenza and Pseudomonas aeruginosa. The use of adjunctive corticosteroid therapy is controversial in the treatment of Pseudomonas aeruginosa corneal ulcers. Atropine is an adjunct therapy that can be given as drops or ointment (1\%) on corneal ulcers to prevent ciliary contractions, reduce pain and synechiae. ${ }^{[11],[12],[13]}$

From several studies, the main antibiotics used for Pseudomonas aeruginosa are aminoglycosides(gentamicin, tobramycin, amikacin, netilmicin), arbapenems (imipenem, meropenem), cephalosporins (ceftazidime, cefepime), fluoroquinolones (ciprofloxacin, levofloxacin with -inhibitors), penicillin with -inhibitors, -lactamases (BLI) (ticarcillin and piperacillin in combination with clavulanic acid or tazobactam), monobactam(aztreonam), Fosfomycin, and polymyxin (colistin, polymyxin B). ${ }^{[14]}$ Sikha Gupta et al. ${ }^{[15]}$ stated that Gatifloxacin showed significant activity against pseudomonal strains followed by ciprofloxacin and tobramycin. The study also reported that Pseudomonas aeruginosa was sensitive to the cephalosporin group, namely ceftazidime (39\%), cefuroxime (37\%), and cefazolin (32\%). A report from the UK has found Pseudomonas aeruginosa to be $100 \%$ sensitive to moxifloxacin and $99 \%$ to ciprofloxacin.

Medical therapy, in this case, includes the administration of topical antibiotics levofloxacin (fluoroquinolone), fortified cefazolin, and systemic antibiotics with ceftriaxone (cephalosporin), which can have a good effect on corneal ulcer infection due to Pseudomonas aeruginosa. It is because the fluoroquinolone and cefazolin groups can eradicate the Pseudomonas aeruginosa bacteria. Ceftriaxone, which belongs to the cephalosporin group, also shows good activity against Gram-positive and Gram-negative bacteria.

In this case, the corneal ulcer developed into a corneal perforation, and a keratoplasty was planned, but there were limitations-the unavailability of a corneal donor.
Other options have been carried out, such as the surgical management of multilayer AMT and pericardial patch graft with an indication of corneal perforation due to corneal ulcers caused by the Pseudomonas aeruginosa bacteria.

The surgical treatment of corneal ulcers is based on size, location, and cause. Especially in perforated corneal ulcers, surgical intervention is required to close the perforation, repair the chambers of the eye, and restore visual function. Corneal gluing is suitable for corneal perforations of less than $2 \mathrm{~mm}$. Pericardial patch graft is a membrane that has been designed to support, repair, and replace the structure and action of connective tissue and regenerate tissue. ${ }^{[13],[16]}$

AMT can provide structural support in areas of corneal ulceration. AMT is a physical layer substrate suitable for epithelial cell migration, helps epithelialization, inhibits inflammation, and has antimicrobial effects. The use of single-layer AMT may be sufficient to treat ulcers but insufficient for deep stromal thinning and not effective in cases of deep ulcers and perforations. Therefore, multilayer AMT may be performed in patients with deeper ulcers, corneal perforations, or descemetocele. Penetrating keratoplasty is a corneal transplant (grafting) with a full-thickness layer, performed in cases where there is a failure of medical treatment and large corneal perforation.[13],[17],[18]

Potential complications of corneal ulcers include an extension of infection to the sclera, formation of corneal opacities or scar tissue (nebula, macula, leukoma), descemetocele, perforation, ectatic cicatrix, corneal fistula, and endophthalmitis or panophthalmitis. The prognosis of a corneal ulcer depends on the size, location, depth, and etiology of the corneal ulcer and its previous ocular condition. Meanwhile, the prognosis of corneal perforation is endophthalmitis or panophthalmitis and the rupture of intraocular blood vessels (vitreous, choroidal, subretinal or subchoroidal hemorrhages).[19],[20],[21]

It is difficult to perform an eye examination in children, mainly when calculating the size and depth of the corneal ulcer, as it is essential for determining the management and evaluation of the corneal ulcer.

This case report illustrates that concentrations obtained with the topical antibiotic levofloxacin, intravenous ceftriaxone provide good efficacy in Pseudomonas aeruginosa infection. AMT and pericardial patch graft with conjunctival sutures over the graft is also quite effective as an alternative for repairing the corneal perforation due to Pseudomonas aeruginosa infection, although, at the time of the course after the procedure, some complications (lysis of the graft) were found. Penetrating keratoplasty is the most vital management strategy for corneal ulcers because corneal perforation has high morbidity. AMT and pericardial patch graft have proven successful adjunct methods due to the lack of donor corneal tissue. 
Establishing a diagnosis of corneal ulcers due to Pseudomonas aeruginosa in children is a challenge in itself during history-taking and physical examination. Thus, the role of cultural examination is crucial. Corneal ulcers due to Pseudomonas aeruginosa are highly destructive. The levels of infection, diagnostic, and therapeutic problems in pediatric patients may differ from those in adults. Lens extrusion and lysis of pericardial patch grafts serve as an example in this case. Keratoplasty is the definitive treatment for corneal ulcers with perforatio; however, multilayer AMT combined with pericardial patch graft can be used as an alternative therapy to accelerate wound healing, reduce inflammation, and maintain the integrity of the eyeball.

\section{References}

[1] Suwal S, Bhandari D, Thapa P, Shrestha MK, Amatya J. Microbiological profile of corneal ulcer cases diagnosed in a tertiary care ophthalmological institute in Nepal. BMC Ophthalmol 2016;16:209. https://doi.org/10.1186/s12886016-0388-9.

[2] Ung L, Acharya NR, Agarwal T, Alfonso EC, Bagga B, Bispo $\mathrm{PJ}$, et al. Infectious corneal ulceration: A proposal for neglected tropical disease status. Bull World Health Organ 2019;97:854-856. https://doi.org/10.2471/BLT.19.232660.

[3] Chirinos-Saldaña P, Bautista de Lucio VM, HernandezCamarena JC, Navas A, Ramirez-Miranda A, Vizuet-Garcia $\mathrm{L}$, et al. Clinical and microbiological profile of infectious keratitis in children. BMC Ophthalmol 2013;13:54. https:// doi.org/10.1186/1471-2415-13-54.

[4] Hong J, Chen J, Sun X, Deng SX, Chen L, Gong L, et al. Paediatric bacterial keratitis cases in Shanghai: Microbiological profile, antibiotic susceptibility and visual outcomes. Eye (Lond) 2012;26:1571-1578. https://doi.org/10.1038/ eye.2012.210

[5] Nicula C, Szabo I. Complicated corneal ulcer. Case report. Rom J Ophthalmol 2016;60:260-263.

[6] Al Otaibi AG, Allam K, Damri AJ, Shamri A Al, Kalantan H, Mousa A. Childhood microbial keratitis. Oman J Ophthalmol 2012;5:28-31. https://doi.org/10.4103/0974-620X.94763.

[7] Colby K, editor. Corneal Diseases in Children. 1st ed. Manhattan: Springer, Cham; 2017.

[8] Gao N, Kumar A, Yu F-SX. Matrix Metalloproteinase-13 as a target for suppressing corneal ulceration caused by Pseudomonas aeruginosa infection. J Infect Dis 2015;212:116127. https://doi.org/10.1093/infdis/jiv016.

[9] Levin LA, Albert DM. Infectious Keratitis. Ocul. Dis. Mech. Manag. 1st ed., Amsterdam: Elsevier Inc; 2010, p. 49-54.

[10] Michael KB, Rotchford A, Ramaesh K. Conjunctival chemosis as a specific feature of Pseudomonas aeruginosa corneal ulcers. Cornea 2016;35:1182-1184. https://doi. org/10.1097/ICO.0000000000000947.

[11] Oka N, Suzuki T, Ishikawa E, Yamaguchi S, Hayashi N, Gotoh N, et al. Relationship of virulence factors and clinical features in keratitis caused by Pseudomonas aeruginosa. Invest Ophthalmol Vis Sci 2015;56:6892-6898. https://doi. org/10.1167/iovs.15-17556.

[12] Sihota R, Tandon R. Disease of the Cornea. Parsons' Dis. Eye. 21st ed., Amsterdam: Elsevier Inc; 2011, p. 200-205.

[13] Kenia VP, Kenia R V, Pirdankar OH. Diagnosis and management protocol of acute corneal ulcer. Int J Heal Sci Res 2020;10:69-78.

[14] Bassetti M, Vena A, Croxatto A, Righi E, Guery B. How to manage Pseudomonas aeruginosa infections. Drugs Context 2018;7:212527. https://doi.org/10.7573/dic.212527.

[15] Gupta S, Mittal S, Nayak N, Satpathy G, Khokhar S, Agarwal T. In vitro antibiotic susceptibility of Pseudomonas aeruginosa corneal ulcer isolates. Ocul Immunol Inflamm 2015;23:252-255. https://doi.org/10.3109/09273948.2014 .883545 .

[16] Alio JL, Rodriguez AE, Martinez LM. Bovine pericardium membrane (tutopatch) combined with solid plateletrich plasma for the management of perforated corneal ulcers. Cornea 2013;32:619-624. https://doi.org/10.1097/ ICO.0b013e31825a6d9a.

[17] Yokogawa H, Kobayashi A, Yamazaki N, Masaki T, Sugiyama K. Surgical therapies for corneal perforations: 10 years of cases in a tertiary referral hospital. Clin Ophthalmol 2014;8:2165-2170. https://doi.org/10.2147/OPTH.S71102.

[18] Abdulhalim B-EH, Wagih MM, Gad AAM, Boghdadi G, Nagy RRS. Amniotic membrane graft to conjunctival flap in treatment of non-viral resistant infectious keratitis: A randomized clinical study. Br J Ophthalmol 2015;99:59-63. https://doi.org/10.1136/bjophthalmol-2014-305224.

[19] Morita Y, Tomida J, Kawamura Y. Responses of Pseudomonas aeruginosa to antimicrobials. Front Microbiol 2014;4:422. https://doi.org/10.3389/fmicb.2013.00422.

[20] Zuorro A, Fidaleo M, Lavecchia R. Solubility enhancement and antibacterial activity of chloramphenicol included in modified $\beta$-cyclodextrins. Bull Korean Chem Soc 2020;31:3460-3462. https://doi.org/10.5012/ BKCS.2010.31.11.3460.

[21] Ranch K, Patel H, Chavda L, Koli A, Maulvi F, Parikh RK. Development of in situ ophthalmic gel of dexamethasone sodium phosphate and chloramphenicol: A viable alternative to conventional eye drops. J Appl Pharm Sci 2017;7:101-108. https://doi.org/10.7324/JAPS.2017.70316. 